\title{
Rare Case of Mediastinal Synovial Sarcoma in an Indonesian Woman
}

\author{
Yuliza $^{1}$, Laksmi Wulandari ${ }^{2}$ \\ ${ }^{I}$ Resident, Department of Pulmonology and Respiratory Medicine, Faculty of Medicine, Universitas Airlangga-Dr. \\ Soetomo General Academic Hospital, Surabaya, Indonesia, ${ }^{2}$ Association Professor, Thoracic Oncology Division, \\ Department of Pulmonology and Respiratory Medicine, Faculty of Medicine, Universitas Airlangga-Dr. Soetomo \\ General Academic Hospital, Surabaya, Indonesia
}

\begin{abstract}
Background: Synovial sarcoma mediastinum is a rare tumor, with a few cases having been reported.

Case Presentation: A 27-year-old Indonesian woman had a complaint of shortness of breath and left chest pain. Chest X-ray showed a massive pleural effusion with reddish-yellow pleural fluid. Thorax CT scan showed a clear demarcated mass in the anterior mediastinum until left medius, with value of lactic acid dehydrogenase of $561 \mathrm{U} / \mathrm{L}$. The patient was diagnosed with mediastinal tumor and performed left anterolateral thoracotomy with tumor weight of $2.354 \mathrm{mg}$. Tumor histopathology consisted of positive Vimentin, BCL2 and CD99 antibodies. The patient performed 3 cycles of chemotherapy after surgery using doxorubicin, ifosfamide, and mesna. Post-chemotherapy results of CT showed metastases in the left hemidiaphragm with an expansion of mass defects and lymphadenopathy in the upper right paratracheal.
\end{abstract}

Conclusion: The prognosis synovial sarcoma mediastinum is worse than synovial sarcoma in other organs as seen in most recurrent cases.

Keywords: Synovial sarcoma mediastinum, malignant tumor, poor prognosis.

\section{Introduction}

Mediastinal synovial sarcoma is a rare neoplasm, with only a few cases having been reported. It has overlapping histological and immunophenotypic features with other tumors in the differential diagnosis, and comprise $<1 \%$ of malignant neoplasm and $<0.01 \%$ of all malignant thoracic neoplasms ${ }^{(1)}$. This translocation is the gold standard for diagnosing synovial sarcoma, where $100 \%$ of the biphasic subtype and $96 \%$ of monophasic

\section{Corresponding Authors:}

\section{Laksmi Wulandari}

Association Professor, Thoracic Oncology Division, Department of Pulmonology and Respiratory Medicine, Faculty of Medicine, Universitas Airlangga-Dr.

Soetomo General Academic Hospital, Surabaya, Indonesia

e-mail: laksmi.wulandari@fk.unair.ac.id subtype show this translocation ${ }^{(2)}$. Mediastinal synovial sarcoma is a malignant tumor that can invade organs that are close to or metastasize to more distant organs ${ }^{(3)}$. In some literatures, therapeutic modalities for mediastinal synovial sarcoma include surgery, chemotherapy and radiation $^{(4)}$. The prognosis of this neoplasm depends on location, size, stage, histology, tumor resection, and metastasis ${ }^{(5)}$. Based on the description above we report a rare case of mediastinal synovial sarcoma in a 27 -yearold Indonesian woman.

Case Presentation: A 27-year-old Indonesian woman presented intermittent shortness of breath since the past 4 months. She also complained of coughing with white phlegm for 1 month, intermittent fever from 3 weeks and left chest pain 6 months before being admitted to hospital. There was no history of haemoptysis or weight loss. The patient had undergone breast tumor surgery and thyroid surgery 8 months and 5 years before hospitalization. Physical examination did not reveal anything significant. Chest X-ray examination 
showed homogeneous opacity in the entire left lung, chest markers X-ray ultrasound was performed for later puncture, and obtained reddish-yellow fluid. Thoracic computerized tomography (CT) scan with contrast revealed a left anterior-medial mediastinal mass measuring $11.95 \times 16.79 \times 24.45 \mathrm{~cm}$ with a necrotic area and multiple punctate calcification with suspicion of a teratoma with a differential diagnosis of lymphoma (Figure 1). Tumor marker examinations showed Lactic Acid Dehydrogenase (LDH) as much as $561 \mathrm{U} / \mathrm{L}$, but Beta human chorionic gonadotropin $(\beta-\mathrm{HCG})$ and Alpha-fetoprotein (AFP) levels were within normal limit. Bone survey examination and upper lower abdominal ultrasound did not show any metastases.

The patient performed explorative thoracotomy with wedge resection for diagnosis. Tumor tissue was obtained with a total weight of $2.354 \mathrm{mg}$ (Figure 2). Macroscopic examination showed gray-white, partially brownish, partially yellowish tissue had a densely brittle solid density. Microscopic examination revealed proliferation of oval to spindle, pleomorphic, hyperchromatic, narrow cytoplasm cells. Some subnuclei were prominent, mitosis $14 / 10$ high power fields (HPF) and necrotic areas appeared between them. The results of the examination demonstrated a malignant spindle mesenchymal tumor with a differential diagnosis of malignant peripheral nerve sheath tumor and synovial sarcoma. Immunohistochemical examination (IHC) results confirmed a synovial sarcoma (Figure 3).

The patient complained of pain after surgery. Thoracic CT scan evaluation showed metastases process. The patient received adjuvant chemotherapy with doxorubicin, ifosfamide, and mesna for three cycles. During first and second chemotherapy, the patient complained of nausea, vomiting, thrush, and grade-2 leukopenia. In the third cycle of chemotherapy, the patient complained of cancer sores, nausea, vomiting and grade- 3 anaemia. The patient received intravenous injection of $4 \mathrm{mg}$ ondansetron, subcutaneous injection of $250 \mathrm{mcg}$ leucogen, and nystatin drop to treat the side effects. Two weeks after the third cycle of chemotherapy, the patient underwent thoracic $\mathrm{CT}$ scan evaluation that showed aggressive metastases process. She declined further treatment and died 3 months later.

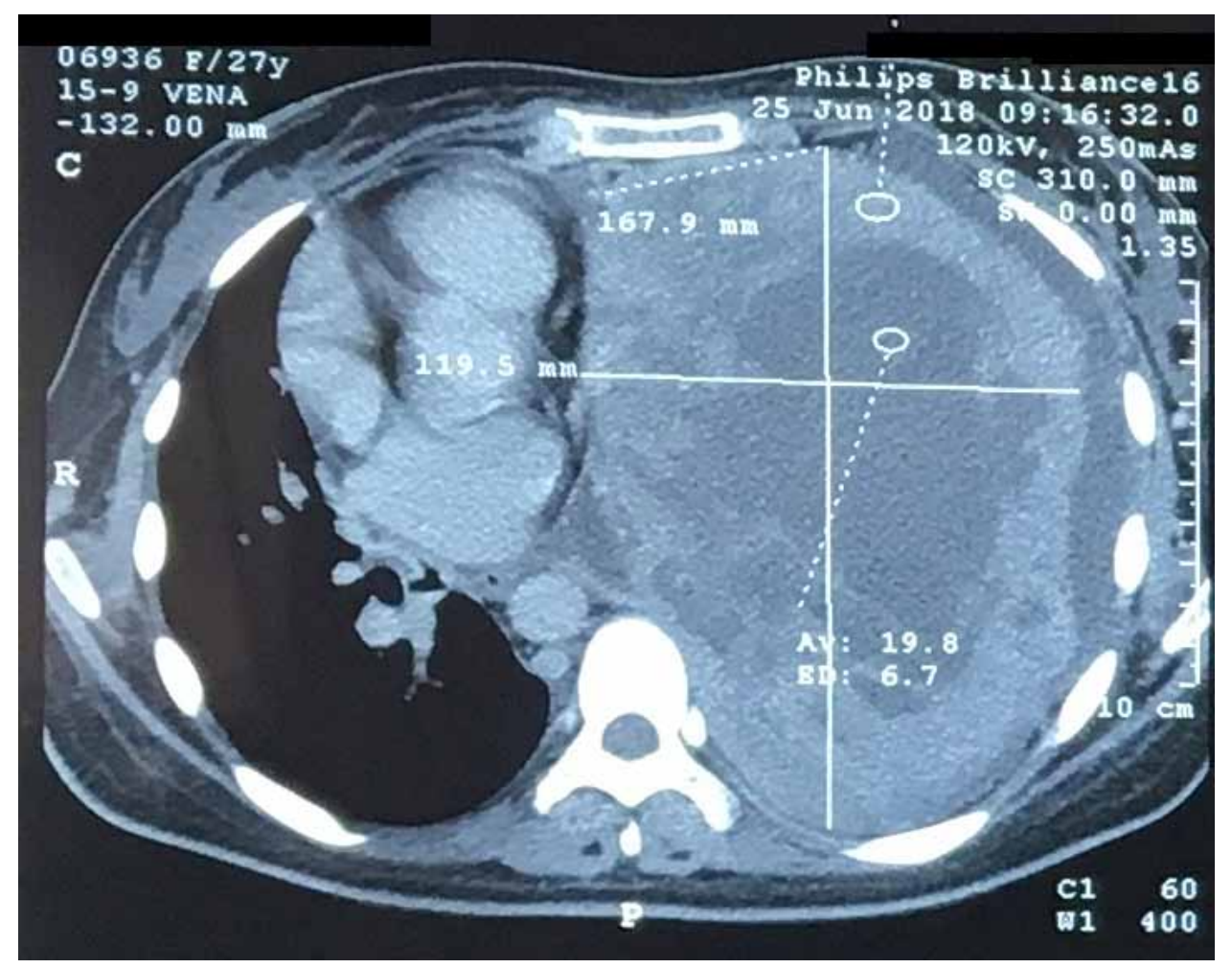

Figure 1. Thoracic CT CT scan with contrast 


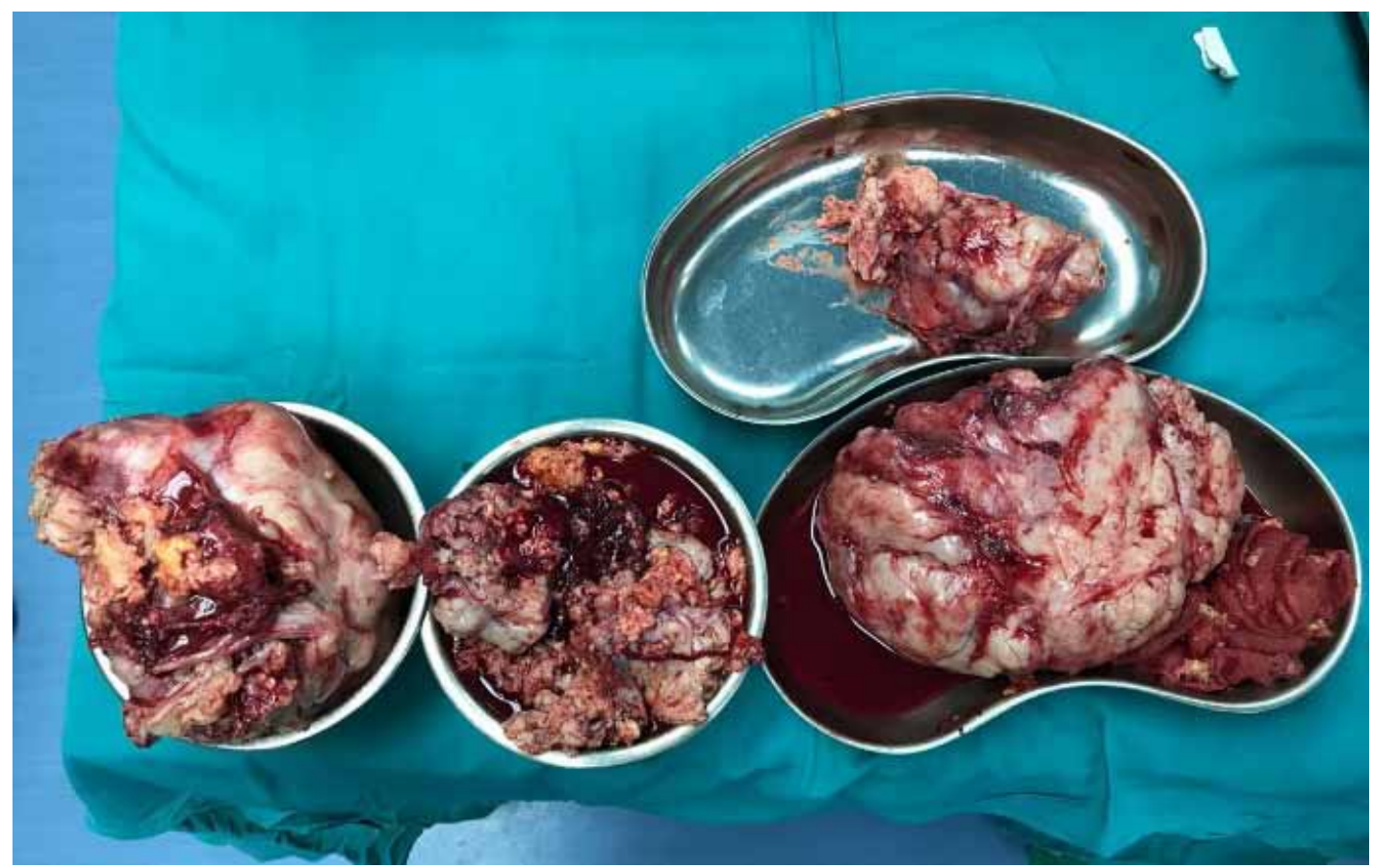

Figure 2. Mediastinal tumor tissue of the patient weighing at $2.354 \mathrm{mg}$
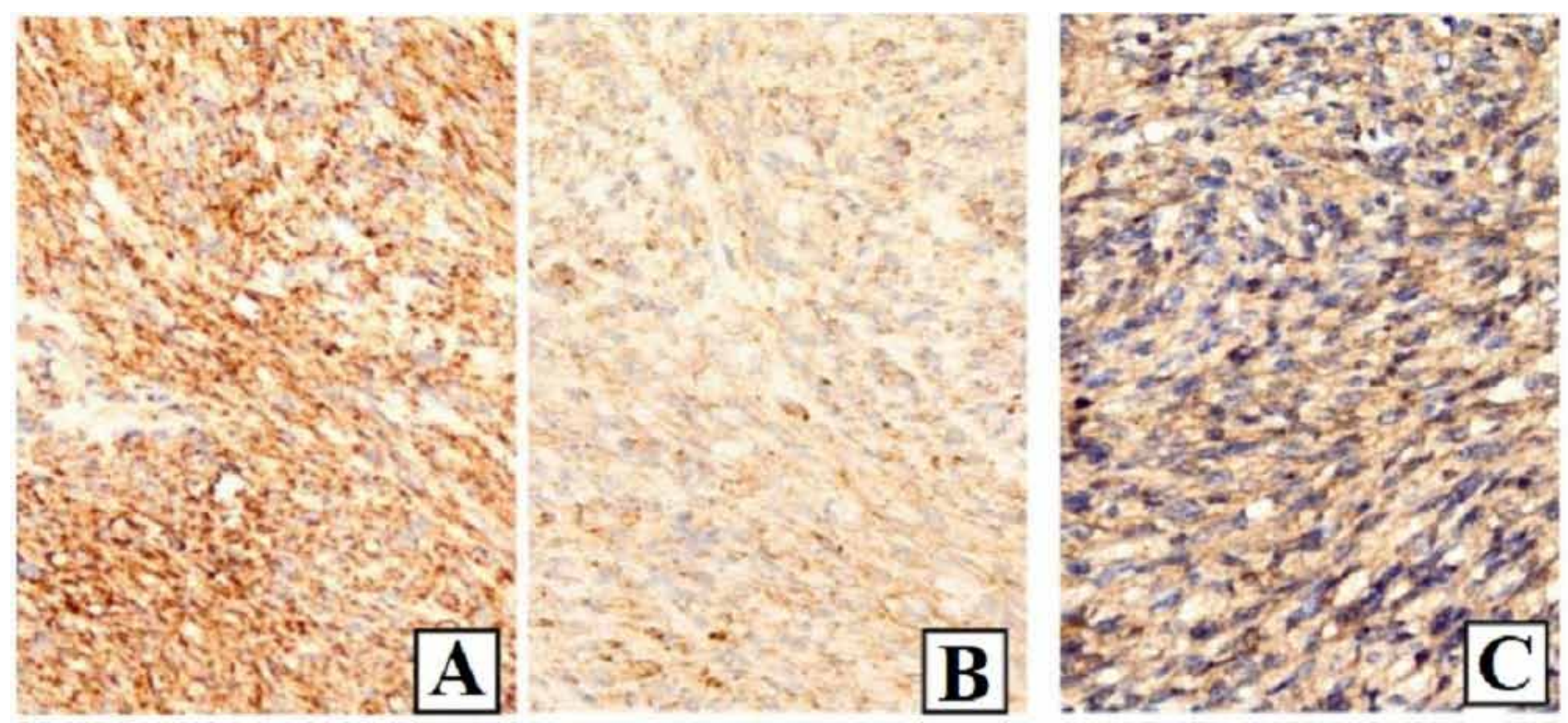

Figure 3. Positive immunohistochemical results in the cytoplasm of tumor cells with antibodies Vimentin (A), BCL2 (B), and CD99 (C).

\section{Discussions}

Mediastinal synovial sarcoma is a rare and aggressive malignant soft tissue tumor occuring most commonly in male and young adults. Only few case reports and series of primary synovial sarcoma are described in the literature so far. Witkin et al. in 1989 reported 4 cases of biphasic mediastinal synovial sarcoma. The patients were all adult males and the tumor frequently adherent to the adjacent pleura or pericardium but none were arising from them. Suster and Moran described 15 cases of primary synovial sarcoma, 9 of their cases presented with anterior mediastinal masses with chest pain and shortness of breath. Thoracic CT scan demonstrates a well-defined homogenous or heterogeneously enhancing mass containing necrotic areas and soft tissue components. Peripheral calcifications were described in 
some cases. In advanced stages, invasion and infiltration of surrounding tissue, and ipsilateral pleural effusion may be presented ${ }^{(6)}$.

Synovial sarcoma is morphologically classified into three subtypes, namely monophasic, biphasic, and poorly differentiated. The monophasic type is dominated by spindle cells, the biphasic type contains epithelial cells and spindle cells, while the poorly differentiated type contains large variants of epithelial cells and spindle cells ${ }^{(5)}$. Tissue specimen of our case morphology showed proliferation of round nucleated, oval to spindle, pleomorphic, hyperchromatic cells, narrow cytoplasm with several prominent subnuclei, and 14/10 HPF mitosis that revealed monophasic type. Immunohistochemical examination of synovial sarcoma was found to be positive for epithelial membrane antigen BCL-2, CD99, S-100, and Vimentin ${ }^{(2)}$. S100 and EMA antibodies were found negative, thus further examination of cytogenetic analysis examination is needed to confirm the presence of chromosome $\mathrm{t}(\mathrm{x} ; 18)(\mathrm{p} 11 ; \mathrm{q} 11)$ translocation that is found in $90 \%$ of synovial sarcoma cases ${ }^{(7)}$.

Mediastinal synovial sarcoma in patient was at stage IV according to the 8th edition of the American Joint Committee on Cancer (AJCC). The classification of soft tissue sarcoma is based on histological profile $(\mathrm{G})$, tumor size $(\mathrm{T})$, lymph node enlargement $(\mathrm{N})$, and metastasis (M). Synovial sarcoma histological profile adapted from the Federation Nationale des Centers de Lutte Contre Le Cancer (FNCLCC) consists of three parameters, namely differentiation, mitotic activity, and the expansion of the necrotic area ${ }^{(8,9)}$.

Mediastinal synovial sarcoma therapy includes surgery, radiotherapy and chemotherapy ${ }^{(3)}$. Complete resection of tumor tissue is a standard management related to survival rates. Synovial sarcoma has a moderate sensitivity to chemotherapy regimens from alkylating agent group consisting of doxorubicin and ifosfamide with varying degrees of response from 30 to $55 \%$. Neoadjuvant chemotherapy with doxorubicin and ifosfamide in mediastinal synovial sarcoma can reduce the size of a large tumor, making it easier for surgery. The most common side effects of chemotherapy from alkylating agent group are nausea, vomiting, leukopenia, and thrombocytopenia. Haemorrhagic cystitis can occur in ifosfamide chemotherapy. To reduce these side effects, medical experts can provide adequate fluid hydration and additional mercaptoethanesulfonate (mesna) therapy according to the patient's weight ${ }^{(2)}$. In several studies, a combination of surgery and radiotherapy showed significant outcome for the survival rate of a patient with high-grade synovial sarcoma. Radiotherapy can be given before surgery, after surgery, or in these two periods using the External Beam Radiation Therapy (EBRT) method ${ }^{(10)}$.

Prognosis of mediastinal synovial sarcoma is worse than synovial sarcoma in other organs, as seen in most cases that often experience recurrence. The prognosis of this tumor is estimated at $35.7 \%$ for the 5 -year survival rate. Factors causing poor clinical and microscopic prognosis include age more than 20 years, male patient, incomplete resection, tumor size more than $5 \mathrm{~cm}$, neurovascular infiltration, extensive tumor necrosis, high rates of mitotic features ( $>10 / 10 \mathrm{HPF}$ ), tumor histology of undifferentiated high grade, biphasic variant, reciprocal SYT-SSXI chromosome translocation, and the presence of distant metastases $(7,11)$. The difference between mediastinal synovial sarcoma and tumor of thymic origin is radiological results. The radiological results of mediastinal synovial sarcoma are located in the lung organs and mediastinal organs while the tumor of thymic origin has no specificity ${ }^{(12)}$.

\section{Conclusion}

Mediastinal synovial sarcoma is a rare case of malignancy in lung tissue. Management includes surgery, radiotherapy, and chemotherapy. The prognosis is quite poor in this reported case.

\section{Funding: None.}

Conflict of Interest: The authors declare that they have no conflict of interest.

Statement of Ethics: The present case report adhered to the Declaration of Helsinki. Informed consent for publication was obtained from the patient.

\section{References}

1. Godil A, Mallick MSA, Khetpal A, Godil FJ, Adam AM, Mallick MJ. Primary Synovial Sarcoma of the Mediastinum in an 18-year old male: A case report. JPMA The Journal of the Pakistan Medical Association. 2018;68(11):1708-10.

2. DeVita VT, Lawrence TS, Rosenberg SA. Cancer of the skin: cancer: principles \& practice of oncology: Lippincott Williams \& Wilkins; 2015.

3. Chatterjee AS, Kumar R, Purandare N, Jiwnani 
S, Karimundackal G, Pramesh CS. Management of locally advanced primary mediastinal synovial sarcoma. Lung India. 2017;34(2):185-8.

4. Ershadi R, Rahim M, Davari H. Primary mediastinal synovial sarcoma: A rare case report. Int J Surg Case Rep. 2016;27:169-71.

5. SinghD, Gilbert S, Natrajan S, Mehta SS, Sivakumar MK, Ramachandra S. Giant Meditational Biphasic Synovial Sarcoma - A Rare Clinical Presentation. Journal Respiratory Disorder. 2016;1(1):103.

6. Souissi OM, Koubaa MK, Boussetta M. Primary Synovial Sarcoma of the Mediastinum: A Case Report and Review of the Literature. Journal of Clinical Case Reports. 2015;05.

7. Abu-Zaid A, AlNajjar A, Alotaibi S, Alshawaf R, Alqeshtaini N, Alhaidar R, et al. Huge primary mediastinal synovial sarcoma fully occupying the right hemithorax. Journal of Cancer Research and Therapeutics. 2018;14(3):682-6.

8. AJCC Cancer Staging System. $8^{\text {th }}$ ed: American Joint Committe on Cancer; 2017.

9. Longo D. Harrison's hematology and oncology. 3 rd ed: McGraw-Hill Education; 2017.

10. Soomro NH, Junejo S, Zafar AA, Siddiqi S. Mediastinal Synovial Sarcoma. Journal of Surgery Pakistan (International). 2014;19(1).

11. Rea G, Somma F, Valente T, Antinolfi G, Di Grezia G, Gatta G. Primary mediastinal giant synovial sarcoma: A rare case report. The Egyptian Journal of Radiology and Nuclear Medicine. 2015;46(1):912.

12. den Bakker MA, Marx A, Mukai K, Ströbel P. Mesenchymal tumours of the mediastinum--part II. Virchows Arch. 2015;467(5):501-17. 